Pacific Journal of Mathematics

TOTALLY PERIPHERAL 3-MANIFOLDS

G. Bring, Klaus Johannson and Peter Scot i 


\title{
TOTALLY PERIPHERAL 3-MANIFOLDS
}

\section{Matthew Brin, Klaus Johannson and Peter Scott}

\begin{abstract}
We will say that a 3-manifold $M$ is totally peripheral, or TP, if every loop in $M$ is freely homotopic into the boundary $\partial M$ of $M$. In this paper, we show that if $M$ is a compact, orientable, 3-manifold which is TP, then there is a component $F$ of $\partial M$ such that the natural map $\pi_{1}(F) \rightarrow \pi_{1}(M)$ is surjective. In the non-orientable case, this result is almost true but there is essentially one counterexample.
\end{abstract}

This result is surprisingly difficult to prove. It is non-trivial even when $\partial M$ is connected. The result is of some interest in its own right, but it has also been used by Brin and Thickstun in their work on open 3-manifolds whose ends are semi-stable. If $M$ is assumed to be orientable and irreducible as well as being compact and TP, then our conclusion easily implies that $M$ is a compression body, i.e. $M$ consists of $F \times I$ with some 2-handles and 3-handles attached to $F \times 1$. If one assumes that $M$ is orientable and hyperbolic this result has been proved by Marden and Maskit. See the lemma in $\S 2$ of $[\mathbf{M}-\mathbf{M}]$ in the case $\rho=1$. Interestingly, their arguments are analytic and completely different from our arguments.

A natural generalisation of the above is obtained by considering a 3-manifold $M$ with a subsurface $B$ of $\partial M$ such that any loop in $M$ is homotopic into $B$. We will say that such a manifold $M$ is totally peripheral relative to $B$. We show that if $M$ is a compact, orientable 3-manifold and if $B$ is a compact subsurface of $\partial M$ such that $M$ is TP rel. $B$, then there is a component $C$ of $B$ such that the natural map $\pi_{1}(C) \rightarrow \pi_{1}(M)$ is surjective. Our proof uses our earlier results on TP manifolds. In the non-orientable case, there are essentially two counterexamples. This result has also been used by Brin and Thickstun in their work on bounded 3-manifolds with semi-stable ends.

In $\S 1$, we deal with the case of orientable manifolds which are TP, and in $\S 2$, we handle the non-orientable case. In $\S 3$ and $\S 4$, we consider the case of a manifold which is TP rel $B$ using the results of the first two sections.

1. The orientable case. In this section, we prove the following result. 
THEOREM 1.1. If $M$ is a compact, orientable 3-manifold which is totally peripheral (TP), then there is a component $F$ of $\partial M$ such that the natural map $\pi_{1}(F) \rightarrow \pi_{1}(M)$ is surjective.

We will say that a surface $F$ in a 3-manifold $M$ is injective if the natural map $\pi_{1}(F) \rightarrow \pi_{1}(M)$ is injective. We will consider cases where $F$ is a 2 -sphere or is one-sided so that this is not equivalent to saying that $F$ is incompressible.

LEMMA 1.2. Let $M$ be a totally peripheral 3-manifold and let $X$ be a submanifold of the interior of $M$ such that $\partial X$ is injective in $M$. Then $X$ is also totally peripheral.

Proof. Let $\lambda$ be a loop in $X$ and let $\Lambda: S^{1} \times I \rightarrow M$ be a homotopy with $\Lambda_{0}=\lambda$ and $\Lambda_{1}\left(S^{1}\right) \subset \partial M$. We homotop $\Lambda$ transverse to $\partial X$. Thus $\Lambda^{-1}(\partial X)$ consists of circles in $S^{1} \times I$. As $\partial X$ is injective in $M$, we can alter $\Lambda$ so as to remove any circles of $\Lambda^{-1}(\partial X)$ which are null-homotopic in $S^{1} \times I$. Now $\Lambda^{-1}(\partial X)$ must still be non-empty and so the restriction of $\Lambda$ to the appropriate sub-annulus of $S^{1} \times I$ defines a homotopy in $X$ between $\lambda$ and a loop in $\partial X$, as required.

LEMMA 1.3. Let $M$ be a totally peripheral 3-manifold and let $F$ be a closed injective surface embedded in the interior of $M$. Then $F$ is two-sided in $M$ and separates $M$. Further, if $M_{1}$ and $M_{2}$ denote the components of $M$ cut along $F$, then the natural map $\pi_{1}(F) \rightarrow \pi_{1}\left(M_{i}\right)$ is an isomorphism for $i=1$ or 2.

REMARK. If $M$ is compact, orientable, irreducible and TP and $F$ is not $S^{2}$, then the $h$-cobordism theorem [He] implies that $F$ is parallel to a component of $\partial M$.

Proof. First we suppose that $F$ is two-sided in $M$. Now any loop in $M$ can be freely homotoped into $\partial M$. In particular, any loop in $M$ can be homotoped to be disjoint from $F$. It follows that $F$ separates $M$ and that any element of $\pi_{1}(M)=\pi_{1}\left(M_{1}\right) * \pi_{1}(F) \pi_{1}\left(M_{2}\right)$ is conjugate into $\pi_{1}\left(M_{1}\right)$ or $\pi_{1}\left(M_{2}\right)$. Hence the natural map $\pi_{1}(F) \rightarrow \pi_{1}\left(M_{i}\right)$ must be an isomorphism, for $i=1$ or 2 .

If $F$ is one-sided in $M$, we let $N$ denote a regular neighbourhood of $F$ in $M$. As $M$ is TP it follows that $N$ is also TP, by Lemma 1.2. But $\pi_{1}(\partial N)$ is a normal subgroup of $\pi_{1}(N)$ of index two and so $N$ cannot be TP. This contradiction completes the proof of Lemma 1.3. 
The main part of this section is the proof of the following result.

THEOREM 1.4. If $M^{3}$ is compact, orientable, irreducible and TP and if $\partial M$ is injective in $M$, then $M$ must be homeomorphic to the 3-ball or to $\Sigma \times I$, where $\Sigma$ is a closed surface, not $S^{2}$.

Before proving this, we explain how Theorem 1.1 follows.

Proof of Theorem 1.1 assuming Theorem 1.4. Let $M$ be a compact, orientable 3-manifold which is TP. Any compact 3-manifold $M$ is a connected sum of prime manifolds $M_{i}[\mathbf{K n}]$ and Lemma 1.3 shows that all the summands except one are simply connected. Clearly, it suffices to consider the case when $M$ itself is prime. Now $M$ cannot be $S^{1} \times S^{2}$ as $M$ must have boundary. Hence $M$ must be irreducible [He]. If $\partial M$ is injective in $M$, then Theorem 1.4 tells us that $M$ is the 3-ball or $\Sigma \times I$, and, in either case, there is a component $F$ of $\partial M$ that $\pi_{1}(F) \rightarrow \pi_{1}(M)$ is surjective.

If $\partial M$ is not injective, we can find a finite collection of disjoint embedded 2-discs in $M$ such that removing the interior of a regular neighbourhood of these discs from $M$ yields a manifold $X$ with $\partial X$ injective in $M$. Lemma 1.3 shows that each component of $\partial X$ is a 2-sphere or is parallel to a component of $\partial M$. Now the fact that $M$ is TP implies that each component of $X$ is TP, by Lemma 1.2, and so Theorem 1.4 implies that each component of $X$ is a 3-ball or $\Sigma \times I$, where $\Sigma$ is a closed surface. If a component $X_{i}$ of $X$ is homeomorphic to $\Sigma_{i} \times I$, the fact that $\Sigma_{\imath} \times 0$ is parallel to a component $C$ of $\partial M$ implies that $X_{i}$ is a collar neighbourhood of $C$. We deduce that $M$ is obtained from $X$ by attaching 1-handles in such a way that for each component $X_{i}$ of $X$ of the form $\Sigma_{i} \times I$, all the 1-handles are attached to one component of $\partial X_{i}$. If we let $F$ denote the unique component of $\partial X$ which is obtained from the connected sum of all the $\Sigma_{i}$ 's then the natural map $\pi_{1}(F) \rightarrow \pi_{1}(M)$ is surjective, completing the proof of Theorem 1.1.

Now we return to the proof of Theorem 1.4 which we re-state for convenience.

THEOREM 1.4. If $M^{3}$ is compact, orientable, irreducible and TP and if $\partial M$ is injective in $M$, then $M$ must be homeomorphic to the 3-ball or to $\Sigma \times I$, where $\Sigma$ is a closed surface not $S^{2}$.

Proof. The hypotheses on $M$ imply that $M$ is Haken. If $M$ is not the 3-ball, we can find an embedded, two-sided injective surface $F$ in $M$ which 
is also boundary-irreducible but is not parallel to a surface in $\partial M$. It follows from Lemma 1.3 that $F$ cannot be closed. Our first step will be to show that $F$ must be an annulus.

Let $N$ denote the manifold obtained from $M$ by cutting along $F$. Of course, $N$ need not be connected. However, each component of $N$ is Haken. The boundary of $N$ contains two copies of $F$ which we denote $L_{1}$ and $L_{2}$ and we write $L=L_{1} \cup L_{2}$. We let $W$ denote $\partial N$ with the interior of a regular neighbourhood of $\partial L$ removed. Note that $W$ is injective in $N$. We will consider the characteristic submanifold $[\mathbf{J o}][\mathbf{J}-\mathbf{S}]$ of the pair $(N, W)$. Our hypothesis that $M$ is TP implies that given a loop $\lambda$ on $F$, there is a homotopy $\Lambda: S^{1} \times I \rightarrow M$, with $\Lambda_{0}=\lambda$ and $\Lambda_{1}\left(S^{1}\right) \subset \partial M$. We can homotop $\Lambda$ transverse to $F$ and then alter $\Lambda$ until $\Lambda^{-1}(F)$ becomes a union of circles essential in $S^{1} \times I$. We use the incompressibility of $F$ in $M$ to eliminate inessential circles from $\Lambda^{-1}(F)$ and use the boundary-irreducibility of $F$ in $M$ to eliminate any arcs from $\Lambda^{-1}(F)$. We obtain a homotopy $\Lambda: S^{1} \times I \rightarrow M$ with $\Lambda_{0}=\lambda$ and $\Lambda_{1}\left(S^{1}\right) \subset \partial M-\partial F$. Now $\Lambda^{-1}(F)$ cuts $S^{1} \times I$ into sub-annuli. We can further homotop $\Lambda$ so as to eliminate any of these annuli which are homotopic into $F$ fixing their boundary. We conclude that either $\lambda$ is homotopic in $F$ to a component of $\partial F$ or that $\lambda$ lies at one end of an essential (singular) annulus in the pair $(N, W)$.

If $F$ is not an annulus, we can certainly find a loop $\lambda$ on $F$ not homotopic into $\partial F$. The above paragraph shows that if $\lambda_{1}$ and $\lambda_{2}$ are the corresponding loops in $L_{1}$ and $L_{2}$ respectively, then $\lambda_{1}$ or $\lambda_{2}$ lies in the characteristic submanifold $V$ of the pair $(N, W)$. For each $i, V \cap L_{l}$ is an incompressible subsurface of $L_{l}$ (possibly not connected). We claim that $V$ must contain $L_{1}$ or $L_{2}$. A nice way to show this would be to show that there is a loop $\lambda$ on $F$ which is not homotopic into any proper, incompressible subsurface of $F$. However, we do not know of a reference for the existence of such a loop. A much simpler argument which will suffice is to observe that if $\alpha_{1}$ and $\alpha_{2}$ are simple non-peripheral loops on $F$, then there is a loop $\beta$ on $F$ which cannot be homotoped to be disjoint from either $\alpha_{1}$ or $\alpha_{2}$. If $\alpha_{1}$ and $\alpha_{2}$ are disjoint, this is easy. If they intersect, then we choose $\beta$ to represent either $g_{1} g_{2}$ or $g_{1} g_{2}^{-1}$, where $\alpha_{\imath}$ represents $g_{i}$ in $\pi_{1}(F)$. If $V \cap L_{i}$ is a proper subsurface of $L_{l}$, there is a boundary loop $\alpha_{i}$ of $V \cap L_{i}$ which is non-peripheral in $L_{l}$. Hence, if $V$ contains neither $L_{1}$ nor $L_{2}$, we find such loops $\alpha_{1}$ and $\alpha_{2}$ on $F$ and then find a loop $\beta$ on $F$ such that $\beta_{l}$ cannot be homotopic into $V \cap L_{l}$, for $i=1$ and 2 . This contradiction shows that $V$ must have a component $U$ which contains $L_{1}$ or $L_{2}$ or both. 
If $U$ contains $L_{1}$ and $L_{2}$, then $U$ must be a product $L_{1} \times I$ and $M$ must be a bundle over $S^{1}$ with fibre $F$. However, the assumption that $M$ is totally peripheral forces $F$ to be an annulus which contradicts our assumption on $F$. To see this, let $M_{F}$ denote the infinite cyclic regular covering of $M$ with $\pi_{1}\left(M_{F}\right)=\pi_{1}(F)$. Of course, $M_{F}$ is homeomorphic to $F \times \mathbf{R}$. We let $p: M_{F} \rightarrow F$ denote the natural projection. Now let $\lambda$ be any loop on $F$, and let $\Lambda: S^{1} \times I \rightarrow M$ be a homotopy between $\lambda$ and a loop in $\partial M$. There is a lift $\bar{\Lambda}$ of $\Lambda$ into $M_{F}$ and $p \circ \bar{\Lambda}: S^{1} \times I \rightarrow F$ is a homotopy between $\lambda$ and a loop in $\partial F$. As $\lambda$ was any loop on $F$, we deduce that $F$ is an annulus as claimed.

We will suppose that $U$ contains $L_{1}$ and not $L_{2}$. Now $U$ must be an $I$-bundle with $L_{1}$ being a component of the $\partial I$-bundle. We note that $F$ separates $M$ and that $U$ is one of the components of $N$. If $U$ is a trivial $I$-bundle, then $F$ is a parallel to a subsurface of $\partial M$ contradicting our choice of $F$. If $U$ is a non-trivial $I$-bundle, then there is a loop in $U$ which cannot be homotoped into $\partial M$, as in the proof of Lemma 1.3. This contradicts our hypothesis that $M$ is TP.

We conclude from the preceding paragraphs that $F$ must be an annulus. Now we can show that $M$ must be a product $\Sigma \times I$. The fact that $M$ is TP implies that the pair $(N, L)$ satisfies a condition which we call relatively TP. We will prove this in Lemma 1.5 below.

Definition. A pair $(N, L)$ is relatively $T P$ if any arc in $(N, L)$ is homotopic in $(N, L)$ into $(\overline{\partial N-L}, L)$.

As this point, we will assume that $F$ is non-separating so that $N$ is connected. We consider the case when $F$ separates $M$ at the end of our proof. Let $\tilde{N}$ denote the universal covering of $N$ and let $\tilde{L}$ denote the pre-image of $L$. Then the pair $(\tilde{N}, \tilde{L})$ is also relatively TP. As $L$ and $\partial N-L$ are injective surfaces in $N$ it follows that each component of $\tilde{L}$ is an infinite strip homeomorphic to $\mathbf{R} \times I$ and that each component of $\partial \tilde{N}-\tilde{L}$ is simply connected. Choose a component $C$ of $\tilde{L}$. As $C$ has only two boundary components, $C$ can meet only two components $X$ and $Y$ of $\bar{\partial} \tilde{N}-\tilde{L}$. Possibly $X$ and $Y$ are equal.

Let $g$ be any element of $\pi_{1}(N)$ and consider its action on $\tilde{N}$. Let $\lambda$ be a path in $\tilde{N}$ joining $C$ to $g C$. As $(\tilde{N}, \tilde{L})$ is relatively TP, we know that $\lambda$ is homotopic in $(\tilde{N}, \tilde{L})$ to a path $\mu$ in $(\overline{\partial \tilde{N}-\tilde{L}}, \tilde{L})$. Thus $\mu$ lies in one

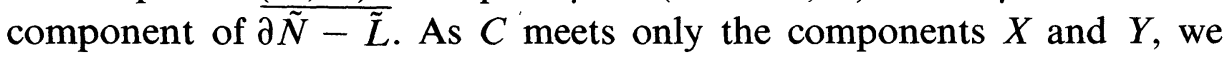
deduce that $\mu$ lies in $X$ or $Y$. Also as $g C$ meets only the components $g X$ and $g Y$, we deduce that $\mu$ lies in $g X$ or $g Y$. Hence we must have one of $X$ 
or $Y$ equal to one of $g X$ or $g Y$. It follows that $\pi_{1}(N)$ can be expressed as a union of cosets of $\operatorname{Stab}(X)$ and $\operatorname{Stab}(Y)$, with at most four cosets being used. Now a theorem of Neumann [N] asserts that if a group $G$ can be expressed as a finite union of cosets of subgroups $C_{1}, \ldots, C_{n}$ of $G$, then some $C_{l}$ has finite index in $G$. Suppose that $\operatorname{Stab}(X)$ is of finite index in $\pi_{1}(N)$ and let $K$ denote the image of $X$ in $\partial N$. Then either $\pi_{1}(N) \cong Z$ or the pair $(N, K)$ is homeomorphic to $(K \times I, K \times 0)$ or to $(V \tilde{\times} I, V \tilde{\times} \partial I)$, where $V$ is a surface and $V \tilde{\times} I$ denotes a non-trivial $I$-bundle over $V$. (See, for example, Theorem 10.5 of [He].) If $\pi_{1}(N) \cong Z$, then the fact that any loop in $N$ is homotopic into $\partial N-L$ implies that the pair $(N, K)$ is again homeomorphic to $(K \times I, K \times 0)$. In each case, it follows that $M$ is an $I$-bundle over some surface $\Sigma$. As $\partial M$ is incompressible, $\Sigma$ must be closed, and the fact that $M$ is TP implies that this $I$-bundle is trivial, by Lemma 1.3. Hence $M$ is $\Sigma \times I$ as required.

At this point, we have completed the proof of Theorem 1.4 when $F$ is non-separating in $M$. If $F$ separates $M$, we apply the above argument to the two components of $N$ and show that each component is an $I$-bundle. Then it follows that $M$ is also an $I$-bundle and hence must be $\Sigma \times I$. This completes the proof of Theorem 1.4, apart from providing the promised proof that the pair $(N, L)$ is relatively TP.

LEMMA 1.5. Let $M$ be a totally peripheral 3-manifold and let $F$ be an embedded two-sided surface in $M$ which is incompressible and boundary irreducible. Let $N$ denote the manifold obtained from $M$ by cutting along $F$ and let $L$ denote the union of the two copies $L_{1}$ and $L_{2}$ of $F$ in $\partial N$.

Then the pair $(N, L)$ is relatively $\mathrm{TP}$.

REMARK. Note that $N$ need not be connected.

Proof. We let $\lambda$ be a path in $(N, L)$.

Case 1. The ends of $\lambda$ lie in distinct components of $L$.

In this case, $F$ must be non-separating and $N$ is connected. We homotop the ends of $\lambda$ in $L$ until $\lambda$ gives a loop $\bar{\lambda}$ in $M$. As $M$ is TP, $\bar{\lambda}$ is homotopic into $\partial M$. So we have a homotopy $\Lambda: S^{1} \times I \rightarrow M$ with $\Lambda_{0}=\bar{\lambda}$, and $\Lambda_{1}\left(S^{1}\right) \subset \partial M$. As before, we can use the incompressibility of $F$ in $M$ to remove any inessential circles from $\Lambda^{-1}(F)$ and we can also use the boundary-irreduciblity of $F$ in $M$ to remove any arcs from $\Lambda^{-1}(F)$ which have both endpoints in $S^{1} \times 1$. As $\Lambda_{0}^{-1}(F)$ is a single point, it follows that $\Lambda^{-1}(F)$ must be a single arc joining $S^{1} \times 0$ to $S^{1} \times 1$. Now $\Lambda$ yields the required homotopy of $\lambda$ into $(\overrightarrow{\partial N-L}, L)$. 
Case 2. The ends of $\lambda$ lie in one component $L_{1}$ of $L$.

In this case, $F$ may or may not separate $M$. We proceed very much as in Case 1. First, note that if $\lambda$ is homotopic in $(N, L)$ into $L_{1}$, then it is certainly homotopic into $(\overline{\partial N-L}, L)$. Hence we can assume that $\lambda$ is not homotopic into $L_{1}$. Now $\lambda$ by itself cannot yield a loop in $M$ of the correct type. So we chose a path $\mu$ in $\left(N, L_{2}\right)$ which is not homotopic into $L_{2}$. We let $\bar{\lambda}$ denote the loop $\lambda \cup \mu$. As in Case 1, we can obtain a homotopy $\Lambda: S^{1} \times I \rightarrow M$ with $\Lambda_{0}=\bar{\lambda}, \Lambda_{1}\left(S^{1}\right) \subset \partial M$ and $\Lambda^{-1}(F)$ contains no inessential circles and no arcs with both endpoints in $S^{1} \times 1$. Now $\Lambda^{-1}(F)$ cannot contain an arc with both endpoints on $S^{1} \times 0$, because then $\lambda$ or $\mu$ would be homotopic into $L_{1}$ or $L_{2}$. Hence $\Lambda^{-1}(F)$ must consist of two arcs joining $S^{1} \times 0$ to $S^{1} \times 1$. As before, $\Lambda$ now yields the required homotopy of $\lambda$ into $(\overline{\partial N-L}, L)$. This completes the proof of Lemma 1.5.

2. The Non-orientable case. In this section we will prove the analogue of Theorem 1.1 for the nonorientable case. There is essentially only one exceptional case and we describe this before stating our precise result.

EXAmple 2.1. Let $T$ denote the torus $S^{1} \times S^{1}$. Thus we can give coordinates $\left(z_{1}, z_{2}, t\right)$ to a point of $T \times I$, where $z_{1}$ and $z_{2}$ are unit complex numbers and $t$ is real, $0 \leq t \leq 1$. Let $\tau: T \times I \rightarrow T \times I$ be the involution given by $\tau\left(z_{1}, z_{2}, t\right)=\left(\bar{z}_{1}, \bar{z}_{2}, 1-t\right)$. Thus $\tau$ has four fixed points. Let $\tilde{M}$ denote the manifold obtained from $T \times I$ by removing the interiors of four disjoint 3-balls centred on the fixed points of $\tau$ and let $M$ denote the manifold $\tilde{M} / \tau$. We claim that $M$ is totally peripheral, but there is no component $F$ of $\partial M$ with $\pi_{1}(F) \rightarrow \pi_{1}(M)$ surjective.

By construction, $\partial M$ consists of a torus $T$ and four projective planes, and $\pi_{1}(T)$ is a subgroup of $\pi_{1}(M)$ of index two. So clearly there is no component $F$ of $\partial M$ with $\pi_{1}(F) \rightarrow \pi_{1}(M)$ surjective. To see that $M$ is totally peripheral, one simply needs to know that every element of $\pi_{1}(M)-\pi_{1}(T)$ has order two and that these elements fall into four conjugacy classes. If we let $u$ denote an element of $\pi_{1}(M)-\pi_{1}(T)$ of order two and $g$ denote any element of $\pi_{1}(T)$, the $g u$ also has order two. This is because $u^{-1} g u=g^{-1}$ by construction of our involution $\tau$. One can also show easily that $g u$ is conjugate to $u$ if and only if $g$ is a square in $\pi_{1}(T)$, so that if $a$ and $b$ form a basis of $\pi_{1}(T)$, the conjugacy classes of elements of order two in $\pi_{1}(M)$ are represented by $t, a t, b t$ and abt.

Now we can state our result in the non-orientable case.

THEOREM 2.2. Let $M$ be a compact 3-manifold which is totally peripheral. Then either there is a component $F$ of $\partial M$ such that the natural map 
$\pi_{1}(F) \rightarrow \pi_{1}(M)$ is surjective or $M$ is of the form $\left(M_{1} \# M_{2}\right) \cup M_{3}$, where $M_{1}$ is the manifold described in Example 2.1, $M_{2}$ is simply connected, and each component of $M_{3}$ is homotopy equivalent to $P^{2} \times I$ and is attached by one boundary component to $M_{1}$.

Proof. If $M$ is orientable, this result is simply Theorem 2.1. Note that the proofs in $\S 1$ give somewhat more information. The component $F$ of $\partial M$, such that $\pi_{1}(F) \rightarrow \pi_{1}(M)$ is surjective is unique unless $M$ is of the form $(\Sigma \times I) \# M^{\prime}$, where $M^{\prime}$ is simply connected.

Now suppose that $M$ is non-orientable and let $\tilde{M}$ be the orientable double covering with covering translation $\tau$. This manifold is also TP. If there is a unique component $\tilde{F}$ of $\partial \tilde{M}$ such that $\pi_{1}(\tilde{F}) \rightarrow \pi_{1}(\tilde{M})$ is surjective, then we must have $\tau \tilde{F}=\tilde{F}$. Thus $\tilde{F}$ covers a component $F$ of $\partial M$ such that $\pi_{1}(F) \rightarrow \pi_{1}(M)$ is surjective, and we have proved the theorem.

Now suppose that $\tilde{M}$ is of the form $(\Sigma \times I) \# M^{\prime}$, where $M^{\prime}$ is simply connected. Thus $\partial \tilde{M}$ consists of surfaces $\Sigma_{0}$ and $\Sigma_{1}$ and some 2-spheres. If $\tau \Sigma_{0}=\Sigma_{0}$, we again have proved the theorem. So we consider the case when $\tau$ interchanges $\Sigma_{0}$ and $\Sigma_{1}$. Thus $\partial M$ consists of one copy of $\Sigma$ and some 2-spheres and projective planes. As $\pi_{1}(\Sigma)$ is a normal subgroup of $\pi_{1}(M)$, no element of $\pi_{1}(M)-\pi_{1}(\Sigma)$ can be conjugate into $\pi_{1}(\Sigma)$. As $M$ is totally peripheral, we deduce that every element of $\pi_{1}(M)-\pi_{1}(\Sigma)$ has order two. Let $t$ be an element of $\pi_{1}(M)-\pi_{1}(\Sigma)$ and let $g \in \pi_{1}(\Sigma)$. The fact that $g t$ also has order two implies that $\operatorname{tg} t^{-1}=g^{-1}$. Hence conjugation by $t$ of $\pi_{1}(\Sigma)$ induces an automorphism of $\pi_{1}(\Sigma)$ which inverts every element. It follows that $\pi_{1}(\Sigma)$ must be abelian. Hence $\Sigma$ is a torus. Now one can show easily that $M$ is as described in Theorem 2.2.

3. The general result in the orientable case. Recall that if $M$ is a 3-manifold and $B$ is a surface in $\partial M$, then $M$ is totally peripheral relative to $B$, if any loop in $M$ is freely homotopic into $B$. We have results and counter examples which are very similar to those obtained for the absolute case when $B$ equals $\partial M$. The main result is the following.

THEOREM 3.1. Let $M$ be a compact, orientable 3-manifold an let $B$ be a compact subsurface of $\partial M$ such that $M$ is $T P$ rel $B$. Then there is $a$ component $C$ of $B$ such that the natural map $\pi_{1}(C) \rightarrow \pi_{1}(M)$ is surjective.

REMARK. Without loss of generality, we can assume that $B$ is injective in $\partial M$.

We need a preliminary result which corresponds to Lemma 1.2 in the absolute case. 
LEMMA 3.2. Let $M$ be a 3-manifold and $B$ a subsurface of $\partial M$ such that $M$ is $T P$ rel $B$. Let $X$ be a 3-dimensional submanifold of $M$ (whose boundary may meet $\partial M$ ), such that $X \cap B$ is empty and the frontier of $X$ in $M$ is an injective surface embedded in $M$. Then $X$ is $T P$ rel fr $X$.

Proof. This is the same as the proof of Lemma 1.2. If $\lambda$ is a loop in $X$, and $\Lambda$ is a homotopy of $\lambda$ into $B$, we homotop $\Lambda$ transverse to the frontier of $X$ and remove any inessential circles of $\Lambda^{-1}(\mathrm{fr} X)$. Now some sub-annulus of $\Lambda$ defines the required homotopy of $\lambda$ into fr $X$.

As in $§ 1$, we first consider the following special case of Theorem 3.1.

THEOREM 3.3. Let $M$ be a compact, orientable, irreducible 3-manifold and let $B$ be a compact subsurface of $\partial M$, such that $M$ is $T P$ rel $B$ and $B$ is injective in $M$. Then either $M$ is a 3-ball and $B$ equals $\partial M$ or there is $a$ component $C$ of $B$ such that the pair $(M, C)$ is homeomorphic to $(C \times I, C$ $\times 0)$.

Proof. Suppose that $\partial M$ is injective in $M$. As $M$ is TP, Theorem 1.4 shows that $M$ is the 3-ball or is a product $\Sigma \times I$, where $\Sigma$ is closed and not $S^{2}$. In the first case, the result we want is clear, so we assume that $M$ is $\Sigma \times I$. Let $B_{i}$ denote $B \cap \Sigma \times\{i\}$, for $i=0,1$, and let $p: \Sigma \times I \rightarrow \Sigma$ denote the natural projection. As $M$ is TP rel $B$, any loop on $\Sigma$ is homotopic in $\Sigma$ into one of the subsurfaces $p\left(B_{1}\right)$ or $p\left(B_{2}\right)$. It follows that either $p\left(B_{1}\right)$ or $p\left(B_{2}\right)$ equals $\Sigma$. For othewise, we can let $\alpha_{i}$ be a boundary component of $p\left(B_{i}\right)$ and use the fact that there is a loop $\beta$ on $\Sigma$ which cannot be homotoped to be disjoint from $\alpha_{1}$ or $\alpha_{2}$ to obtain a contradiction. Hence either $B_{1}$ or $B_{2}$ is the required component $C$ of $B$.

We are left with the case when $\partial M$ is compressible in $M$. Thus there is a 2-disc $D$ embedded in $M$ which splits $\pi_{1}(M)$ non-trivially as a free product. Note that $\partial D$ cannot lie in $B$ as $B$ is injective in $M$. Note also that $\partial D$ must meet $\partial B$, because there are loops in $M$ which cannot be homotoped to be disjoint from $D$ and yet any loop in $M$ is homotopic into $B$. Now we argue as at the end of the proof of Theorem 1.4. Let $N$ denote $M$ cut along $D$, let $L$ denote the union of the two 2-discs $L_{1}$ and $L_{2}$ in $\partial N$ corresponding to $D$ and let $W$ denote $B \cap \partial N$. Note that $W$ is injective in $N$. Then, as in Lemma 1.5, any arc in $(N, L)$ is homotopic in $(N, L)$ into $(W, W \cap L)$. It is also easy to show that $N$ is TP rel $W$.

At this point, we will assume that $N$ is connected, and will consider the case when $D$ separates $M$ at the end of our proof. Let $\tilde{N}$ denote the 
universal covering of $N$ and let $\tilde{W}$ and $\tilde{L}$ denote the pre-images of $W$ and $L$. Choose a component $L_{0}$ of $\tilde{L}$ and let $X_{1}, \ldots, X_{n}$ denote the components of $\tilde{W}$ which meet $L_{0}$. For any element $g$ of $\pi_{1}(N)$, consider an arc in $\tilde{N}$ joining $L_{0}$ and $g L_{0}$. As any $\operatorname{arc}$ in $(\tilde{N}, \tilde{L})$ is homotopic in $(\tilde{N}, \tilde{L})$ into $(\tilde{W}, \tilde{W} \cap \tilde{L})$, we see that there are integers $i$ and $j$ such that $g X_{i}=X_{j}$. It follows that $\pi_{1}(N)$ is the union of finitely many cosets of the subgroups $\operatorname{Stab}\left(X_{i}\right)$. Hence, by Neumann's result $[\mathbf{N}]$, some $\operatorname{Stab}\left(X_{\imath}\right)$ is of finite index in $\pi_{1}(N)$. Let $K$ denote the component of $W$ in $\partial N$ covered by this $X_{i}$. Then (see, for example, Theorem 10.5 of $[\mathbf{H e}]$ ), either $\pi_{1}(N) \cong \mathbf{Z}$ or the pair $(N, K)$ is homeomorphic to $(K \times I, K \times 0)$ or to $(V \tilde{\times} I, V \tilde{\times} \partial I)$ for some surface $V$. We claim that the pair $(N, K)$ cannot be of the form $(V \tilde{\times} I, V \tilde{\times} \partial I)$. For if this were the case then $W$ would consist of the disjoint union of $K$ and of some annuli parallel to $\partial K$. The fact that $N$ is TP rel $W$ would imply that $N$ is TP rel $K$ and hence that any element of $\pi_{1}(N)$ is conjugate into $\pi_{1}(K)$. As $\pi_{1}(K)$ is a proper normal subgroup of $\pi_{1}(N)$, this is a contradiction. A similar argument shows that if $\pi_{1}(N) \cong \mathbf{Z}$, then $\pi_{1}(K)$ must equal $\pi_{1}(N)$, so that, in all cases, the pair $(N, K)$ is homeomorphic to $(K \times I, K \times 0)$.

Let $K_{1}, \ldots, K_{n}$ denote the components of $W$ such that the natural map $\pi_{1}\left(K_{i}\right) \rightarrow \pi_{1}(N)$ is an isomorphism. Lemma 3.4 below shows that there is an $\operatorname{arc} \lambda$ in $N$ joining $L_{1}$ to $L_{2}$ such that $\lambda$ is not homotopic in $(N, L)$ into any component of $W$ other than $K_{1}, \ldots, K_{n}$. Let $\bar{\lambda}$ denote the corresponding loop in $M$. We know that $\bar{\lambda}$ is homotopic to a loop $\bar{\mu}$ in $B$ and earlier arguments show that this homotopy can be chosen to induce a homotopy of $\lambda$ to an arc $\mu$ of $W$. Our choice of $\lambda$ implies that $\mu$ lies in some $K_{i}$. It follows that when we glue $L_{1}$ to $L_{2}$ to form $M$, some arc of $\partial K_{l}$ is glued to another arc of $\partial K_{i}$. Let $C$ be the component of $B$ which contains this $K_{i}$. Then clearly the natural map $\pi_{1}(C) \rightarrow \pi_{1}(M)$ is surjective. As $B$ is injective in $M$, the relative $h$-cobordism theorem [He] implies that the pair $(M, C)$ is homeomorphic to $(C \times I, C \times 0)$ as required.

Apart from the proof of Lemma 3.4, this completes the proof of Theorem 3.3 on the assumption that $D$ fails to separate $M$. If $D$ separates $M$ into manifolds $N^{\prime}$ and $N^{\prime \prime}$, we let $L^{\prime}$ and $L^{\prime \prime}$ denote the copies of $D$ in $\partial N^{\prime}$ and $\partial N^{\prime \prime}$ respectively and let $W^{\prime}$ and $W^{\prime \prime}$ denote $B \cap N^{\prime}$ and $B \cap N^{\prime \prime}$. The arguments above show that there is a component $K^{\prime}$ of $W^{\prime}$ such that the pair $\left(N^{\prime}, K^{\prime}\right)$ is homeomorphic to $\left(K^{\prime} \times I, K^{\prime} \times 0\right)$, and a component $K^{\prime \prime}$ of $W^{\prime \prime}$ such that the pair $\left(N^{\prime \prime}, K^{\prime \prime}\right)$ is homeomorphic to $\left(K^{\prime \prime} \times I, K^{\prime \prime} \times 0\right)$. It follows that $N$ is a handlebody and hence that we can always find a non-separating disc $D$ in $N$. The final step in the proof of Theorem 3.3 is a proof of the following result. 
LEMMA 3.4. Let $K_{1}$ be a compact surface with non-empty boundary and let $N$ denote $K_{1} \times I$. Let $W$ denote a compact subsurface of $\partial N$, injective in $\partial N$, such that $K_{1}$ is a component of $W$, and let $K_{1}, \ldots, K_{n}$ denote those components of $W$ such that the natural map $\pi_{1}\left(K_{i}\right) \rightarrow \pi_{1}(N)$ is an isomorphism. Let $L_{1}$ and $L_{2}$ be disjoint 2-discs in $\partial N$ such that $L_{i}$ meets $W$ only in some submanifold of $\partial L_{i}$. Let $L$ denote $L_{1} \cup L_{2}$ and suppose that any arc in $(N, L)$ is homotopic in $(N, L)$ into $(W, W \cap L)$. Then there is an arc $\lambda$ in $N$ joining $L_{1}$ to $L_{2}$ such that $\lambda$ is not homotopic in $(N, L)$ into any component of $W$ other than $K_{1}, \ldots, K_{n}$.

REMARK. Note that $n \leq 2$, unless $\pi_{1}(N)$ is trivial or $\mathbf{Z}$.

Proof. First note that if $N$ is simply connected, then $W=\bigcup_{i=1}^{n} K_{i}$, so that the result is trivial. Now suppose that $N$ is not simply connected and choose the product structure on $N$ so that $W-K_{1}$ lies in $K_{1} \times 1$, where $K_{1}$ is identified with $K_{1} \times 0$. Le $p: K_{1} \times I \rightarrow K_{1}$ denote the natural projection. Then there is an essential simple loop $\sigma$ in $K_{1}$ which meets no component of $p(W)$ other than $K_{1}, p\left(K_{2}\right), \ldots, p\left(K_{n}\right)$. Let $V$ denote the annulus $\sigma \times I$ in $K_{1} \times I$. Thus $V$ is an essential annulus in $N$ which meets no component of $W$ other than the $K_{i}$ 's. By choosing $\sigma$ transverse to $\partial L_{1}$ and $\partial L_{2}$, we can ensure that $V \cap L_{i}$ consists of a finite number $n_{i}$ of arcs, for $i=1$ and 2 .

Suppose that any $\operatorname{arc} \lambda$ joining $L_{1}$ to $L_{2}$ is homotopic in $(N, L)$ into a component of $W$ other than $K_{1}, \ldots, K_{n}$, and let $\mu$ denote a loop in $N$. We can homotop $\mu$ so that $\mu$ is the union of two sub-arcs $\mu_{1}$ and $\mu_{2}$ each joining $L_{1}$ to $L_{2}$. Let $\lambda_{1}$ and $\lambda_{2}$ be arcs in $W$ homotopic in $(N, L)$ to $\mu_{1}$ and $\mu_{2}$ and not lying in $K_{1}, \ldots, K_{n}$. Thus, in particular, $\lambda_{i} \cap V$ is empty. The loop $\mu$ in $N$ can be homotoped to be the union of four sub-arcs $\mu_{1}, \mu_{2}$ and $\nu_{1}, \nu_{2}$ where each $\nu_{i}$ lies in $L_{i}$. Further each $\nu_{i}$ can be chosen to meet $V$ in at most $n_{i}$ points. It follows from our hypothesis at the start of this paragraph that any loop in $N$ can be homotoped so as to meet $V$ in at most $\left(n_{1}+n_{2}\right)$ points. But this is impossible, for the essentiality of $V$ in $N$ implies that there is a loop $\mu$ in $N$ which cannot be homotoped disjoint from $V$, and then, for all $n$, the loop $\mu^{n}$ must meet $V$ in at least $n$ points. This contradiction completes the proof of Lemma 3.4.

Now we can prove Theorem 3.1 .

Proof of Theorem 3.1. Again we follow closely the arguments of $\$ 1$. First, it suffices to consider the case of Theorem 3.1 when $M$ is irreducible. Thus if $B$ is injective in $M$, Theorem 3.3 yields component $C$ of $B$ such that $\pi_{1}(C) \rightarrow \pi_{1}(M)$ is surjective, as required. 
If $B$ is not injective in $M$, we can find a finite collection of disjoint embedded 2-discs $D_{i}$ in $M$ such that $\partial D_{i}$ is an essential loop in $B$ and such that if $U$ is a regular neighbourhood of the union of the $D_{i}$ 's, if $X$ denotes $\overline{M-U}$ and if $B_{X}$ denotes $B-(U \cap B)+$ fr $U$, then $B_{X}$ is injective in $X$. Note that $B_{X}$ is a compact subsurface of $\partial X$. If we isotop $X$ slightly in $M$ so that the interior of $B_{X}$ moves into the interior of $M$ and the rest of $\partial X$ is unmoved, we can apply Lemma 3.2 to each component of the resulting submanifold $X^{\prime}$ of $M$. As $\operatorname{fr} X^{\prime}$ is $B_{X}$, it follows that each component $X_{i}$ of $X$ is TP rel $B_{i}$, where $B_{i}=B_{X} \cap X_{i}$. Thus, for each $i$, there is a component $K_{i}$ of $B_{i}$ such that the pair $\left(X_{i}, K_{i}\right)$ is homeomorphic to $\left(K_{i} \times I, K_{i} \times 0\right)$. Now $M$ is formed from the $X_{i}$ 's by attaching 1-handles. Let $Z_{i}$ denote the union of the attaching discs on $\partial X_{i}$, and recall that $Z_{i}$ is contained in $B_{i}$. As $M$ is TP rel $B$, it follows, as in Lemma 1.5, that any arc in $\left(X_{i}, Z_{i}\right)$ is homotopic in $\left(X_{i}, Z_{i}\right)$ into $\left(B_{i}, B_{i} \cap Z_{i}\right)$. A similar argument to that used at the end of the proof of Theorem 3.4 shows that $Z_{i}$ must lie in a component $C_{i}$ of $B_{i}$ such that the natural map $\pi_{1}(C) \rightarrow \pi_{1}\left(X_{i}\right)$ is an isomorphism. If we let $C$ denote the component of $B$ which contains $\bar{C}_{i}-Z_{i}$, for all $i$, then the natural map $\pi_{1}(C) \rightarrow \pi_{1}(M)$ is surjective, completing the proof of Theorem 3.1.

4. The general result in the non-orientable case. In this section, we will prove the analogue of Theorem 3.1 for the non-orientable case. There are essentially only two exceptional cases, one of which was described in Example 2.1. Here is the other exception.

EXAmple 4.1. Let $A$ denote the annulus $S^{1} \times I$ and let $\tau$ be the involution on $A \times I$ given by $\tau(z, s, t)=(\bar{z}, 1-s, 1-t)$. Thus $\tau$ has two fixed points. Le $\tilde{M}$ denote the manifold obtained from $A \times I$ by removing the interiors of two disjoint 3-balls centred on the fixed points of $\tau$ and let $M$ denote the manifold $\tilde{M} / \tau$. Let $B$ denote the union of the annulus $(A \times \partial I) / \tau$ and the two projective plane components of $\partial M$. We claim that $M$ is TP rel $B$, but there is no component $C$ of $B$ with $\pi_{1}(C) \rightarrow \pi_{1}(M)$ surjective.

By construction, the annulus component of $B$ has fundamental group which is of index two in $\pi_{1}(M)$. So clearly there is no component $C$ of $B$ with $\pi_{1}(C) \rightarrow \pi_{1}(M)$ surjective. To see that $M$ is TP rel $B$, one simply needs to observe that $\pi_{1}(M)$ is the infinite dihedral group $D(\infty)$ which is isomorphic to $\mathbf{Z}_{2} * \mathbf{Z}_{2}$. In particular, every element of $\pi_{1}(M)-\pi_{1}(\tilde{M})$ has order two and these elements fall into two conjugacy classes.

Now we can state our result in the non-orientable case. 
THEOREM 4.2. Let $M$ be a compact 3-manifold and let $B$ be a compact subsurface of $\partial M$ such that $M$ is $T P$ rel $B$. Assume that $B$ is injective in $\partial M$. Then either there is a component $C$ of $B$ such that the natural map $\pi_{1}(C) \rightarrow \pi_{1}(M)$ is surjective or $M$ is of the form $\left(M_{1} \# M_{2}\right) \cup M_{3}$, where $M_{1}$ is the manifold described in Example 2.1 or 4.1, $M_{2}$ is simply connected and each component of $M_{3}$ is homotopy equivalent to $P^{2} \times I$ and is attached to $M_{1}$ by one boundary component. Also, in the first case $B$ must contain $\partial M$, and in the second case $B$ must have at least the three components described in Example 4.1.

Proof. If $M$ is orientable, this result is simply Theorem 3.1. Note that it follows from the proofs in $\S 3$, that the component $C$ of $B$ such that $\pi_{1}(C) \rightarrow \pi_{1}(M)$ is surjective is unique unless $(M, C)$ is of the form $\left(C \times I \# M^{\prime}, C \times 0\right)$, where $M^{\prime}$ is simply connected.

Now suppose that $M$ is non-orientable and let $\tilde{M}$ be the orientable double covering with covering translation $\tau$, and let $\tilde{B}$ denote the preimage of $B$. Thus $\tilde{M}$ is TP rel $\tilde{B}$ and so, by Theorem 3.1 , there is a component $\tilde{C}$ of $\tilde{B}$ such that $\pi_{1}(\tilde{C}) \rightarrow \pi_{1}(\tilde{M})$ is surjective. If $\tau \tilde{C}=\tilde{C}$, then $\tilde{C}$ covers a component $C$ of $B$ such that $\pi_{1}(C) \rightarrow \pi_{1}(M)$ is surjective, which proves our theorem at once. Hence we suppose that $\tau \tilde{C} \neq \tilde{C}$, so that $(\tilde{M}, \tilde{C})$ is $\left(\tilde{C} \times I \# M^{\prime}, \tilde{C} \times 0\right)$, where $M^{\prime}$ is simply connected. Thus $\tilde{B}$ consists of $\tilde{C}$, $\tau \tilde{C}$ and some annuli in $\partial(\tilde{C} \times I)$ and some 2 -spheres. Let $C$ denote the image of $\tilde{C}$ in $M$. Then $B$ consists of $C$ together with some annuli, Moebius bands and projective planes. If $C$ is closed, there can be no annuli or Moebius bands in $B$ and the arguments of Theorems 2.2 apply to show that $M$ is of the form $\left(M_{1} \# M_{2}\right) \cup M_{3}$, where $M_{1}$ is the manifold described in Example 2.1, and $M_{2}, M_{3}$ are as described in Theorem 4.2. Clearly $B$ must contain $\partial M_{1}$ as $M$ is TP rel $B$.

If $C$ has boundary, we will show that the other exceptional case occurs. We know that $\pi_{1}(M)$ is an extension of the free group $\pi_{1}(C)$ by $\mathbf{Z}_{2}$ and that any loop in $M$ representing an element of $\pi_{1}(M)-\pi_{1}(C)$ is homotopic into one of the annulus, Moebius band or projective plane components of $B$. Hence $\pi_{1}(M)-\pi_{1}(C)$ contains only a finite number of conjugacy classes of primitive elements of infinite order.

Now $\pi_{1}(M)$ is the fundamental group of a graph $\Gamma$ of groups each of which is a subgroup of $\mathbf{Z}_{2}$ [KPS]. If $\Gamma$ contained a loop $\lambda$ with $\mathbf{Z}_{2}$ attached to $\lambda$, then $\pi_{1}(M)$ would contain $\mathbf{Z} \times \mathbf{Z}_{2}$. Now a theorem of Epstein [E] implies that $M$ must be a connected sum $M_{1} \# M_{2}$ where $\pi_{1}\left(M_{1}\right)$ is $\mathbf{Z} \times \mathbf{Z}_{2}$ and $M_{1}$ is closed. But such a manifold cannot be TP as no essential loop in $M_{1}$ can be homotopic into $\partial M$. Hence $\Gamma$ cannot contain a loop with $Z_{2}$ 
attached. Hence any edge of $\Gamma$ with $\mathbf{Z}_{2}$ attached can be contracted and, by repeating, we conclude that $\Gamma$ can be chosen so that all edge groups are trivial. Thus $\pi_{1}(M)$ is a free product of the form $\left(*_{i=1}^{r} \mathbf{Z}_{2}\right) * F_{n}$, where $F_{n}$ denotes the free group of rank $n$. Note that $\pi_{1}(C)$ is the kernel of a map $\pi_{1}(M) \rightarrow \mathbf{Z}_{2}$ which injects each $\mathbf{Z}_{2}$ subgroup.

We claim that because $\pi_{1}(M)-\pi_{1}(C)$ contains only a finite number of conjugacy classes of primitive elements of infinite order, $\pi_{1}(M)$ must be $\mathbf{Z}, \mathbf{Z}_{2}$ or $\mathbf{Z}_{2} * \mathbf{Z}_{2}$. For if $\pi_{1}(M)$ had three $\mathbf{Z}_{2}$-factors, we denote the generators of the factors by $a, b$ and $c$ and consider the elements $a(b c)^{n}$ of $\mathbf{Z}_{2} * \mathbf{Z}_{2} * \mathbf{Z}_{2}$ to obtain a contradicton. If $\pi_{1}(M)$ had a $\mathbf{Z}$ and a $\mathbf{Z}_{2}$ factor we denote the generators by $a$ and $b$ respectively and consider the elements $a^{2 n} b$ to obtain a contradiction. Finally if $\pi_{1}(M)$ is free with basis $a_{1}, \ldots, a_{n}$, some $a_{i}$ must lie in $\pi_{1}(M)-\pi_{1}(C)$. Hence if $n \geq 2$ and $a_{2}$ lies in $\pi_{1}(M)-\pi_{1}(C)$, we can consider the elements $a_{1}^{2 n} a_{2}$ to obtain a contradiction.

If $\pi_{1}(M)$ is cyclic, it is trivial that there is a component $C$ of $B$ such that $\pi_{1}(C) \rightarrow \pi_{1}(M)$ is surjective. For a loop representing a generator of $\pi_{1}(M)$ can be homotoped into $B$. Hence $\pi_{1}(M)$ must be $\mathbf{Z}_{2} * \mathbf{Z}_{2}$ and it follows easily that $M$ is of the form $\left(M_{1} \# M_{2}\right) \cup M_{3}$ where $M_{1}$ is as described in Example 4.1 and $M_{2}, M_{3}$ are as described in Theorem 4.2.

\section{REFERENCES}

[E] D. B. A. Epstein, Projective planes in 3-manifolds, Proc. London Math. Soc., 11 (1961), 469-484.

[He] J. Hempel, 3-manifolds, Ann. of Math. Studies 86, Princeton University Press, Princeton, New Jersey 1976.

[J-S] W. Jaco and P. B. Shalen, Seifert fibred spaces in 3-manfolds, Memoirs of Amer. Math. Soc., 220 (1979).

[Jo] K. Johannson, Homotopy Equivalences of 3-manifolds With Boundary, Springer Lecture Note 761, (1979).

[KPS] A. Karras, A. Pietrowski and D. Solitar, Finitely generated groups with a free subgroup of finite index, J. Australian Math. Soc., 16 (1973), 458-466.

[Kn] H. Kneser, geschlossene Flächen in dreidimensionale Mannigfaltigkeiten, Jahresbericht der Deutsche Math. Verein., 38 (1929), 248-260.

[M-M] A. Marden and B. Maskit, On the isomorphism theorem for Kleinian groups, Invent. Math., 51 (1979), 9-14.

[N] B. H. Neumann, Groups covered by permutable subsets, J. London Math. Soc., 29 (1954), 236-248.

Received July 1, 1983 and in revised form December 14, 1983. The work in this paper was carried out while the last author was visiting the University of Michigan and the University of Melbourne. He was partially supported by both universities and by NSF grant 81-02469. 
STATE UNIVERSITY OF NeW YORK

BINGHAMTON, NY 13901

USA

UNIVERSITÄTSSTRASSE 1

D-4800 BIELEFELD 16

GERMANY

AND

THE UNIVERSITY

P. O. Box 147

LIVERPOOL, L69 3BX

ENGLAND 



\title{
PACIFIC JOURNAL OF MATHEMATICS EDITORS
}

DONALD BABBITT (Managing Editor)
University of California
Los Angeles, CA 90024
CHARLEs R. DEPrIMA
California Institute of Technology
Pasadena, CA 91125
R. FINN
Stanford University
Stanford, CA 94305

DoNAld BABBitT (Managing Editor)

Hermann FlaschKa

University of Arizona

Tucson, AZ 85721

RAmesh A. Gangolli

University of Washington

Seattle, WA 98195

ROBION KIRBY

University of California

Berkeley, CA 94720

C. C. MOORE

University of California

Berkeley, CA 94720

\author{
Hugo Rossi \\ University of Utah \\ Salt Lake City, UT 84112 \\ H. SAMELSON \\ Stanford University \\ Stanford, CA 94305 \\ HAROLD STARK \\ University of California, San Diego \\ La Jolla, CA 92093
}

ASSOCIATE EDITORS

R. ARENS

E. F. BECKENBACH
(1906-1982)

B. H. NEUMANN

F. WOLF

K. Yoshida

\section{SUPPORTING INSTITUTIONS}

UNIVERSITY OF ARIZONA

UNIVERSITY OF BRITISH COLUMBIA

CALIFORNIA INSTITUTE OF TECHNOLOGY

UNIVERSITY OF CALIFORNIA

MONTANA STATE UNIVERSITY

UNIVERSITY OF NEVADA, RENO

NEW MEXICO STATE UNIVERSITY

OREGON STATE UNIVERSITY
UNIVERSITY OF OREGON

UNIVERSITY OF SOUTHERN CALIFORNIA

STANFORD UNIVERSITY

UNIVERSITY OF HAWAII

UNIVERSITY OF TOKYO

UNIVERSITY OF UTAH

WASHINGTON STATE UNIVERSITY

UNIVERSITY OF WASHINGTON 


\section{Pacific Journal of Mathematics}

\section{Vol. 118, No. $1 \quad$ March, 1985}

Dan Amir, On Jung's constant and related constants in normed linear spaces ...1 Abdul Aziz, On the location of the zeros of certain composite polynomials . . 17 Joseph Barback, On hereditarily odd-even isols and a comparability of

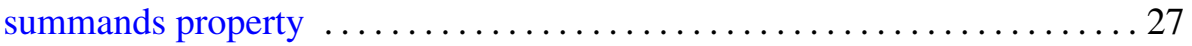

Matthew G. Brin, Klaus Johannson and Peter Scott, Totally peripheral 3-manifolds ........................................ 37

Robert F. Brown, A topological bound on the number of distinct zeros of an

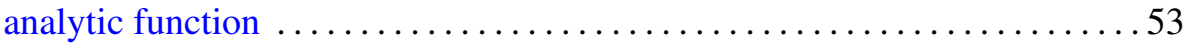

K. C. Chattopadhyay, Not every Lodato proximity is covered .......... 59

Beverly Diamond, Some properties of almost rimcompact spaces . .......63 63

Manfred Dugas and Rüdiger Göbel, On radicals and products . ......... 79

Abdelouahab El Kohen, A hyperbolic problem .................. 105

Harry Gonshor, Remarks on the Dedekind completion of a nonstandard model of the reals ................................... 117

William H. Kazez, On equivalences of branched coverings and their action

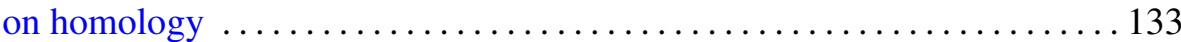

Darrell Conley Kent, On the Wallman order compactification .......... 159

Martin Andrew Magid, Lorentzian isoparametric hypersurfaces . . . . . . 165

Milan Miklavčič, Stability for semilinear parabolic equations with noninvertible linear operator

Richard Dean Neidinger and Haskell Paul Rosenthal, Norm-attainment of linear functionals on subspaces and characterizations of Tauberian operators

Johannes Vermeer, Closed subspaces of $H$-closed spaces 